
\title{
25 Research Square \\ Functional Connectivity Disturbances of the Locus Coeruleus in Chronic Insomnia Disorder
}

Chunlong Li ( $\triangle 960559619 @ q q . c o m)$

Guangzhou Medical College First Affiliated Hospital https://orcid.org/0000-0001-9316-4175

Xiaofen Ma

Guangdong Second Provincial General Hospital

\section{Yuexia Liu}

The Second Affiliated Hospital of Guangzhou University of Chinese Medicine

\section{Ning Yang}

Guangdong Second Provincial General Hospital

\section{Zhihong Lan}

Zhuhai City People's Hospital

\section{Shumei Huang}

Guangdong Second Provincial General Hospital

\section{Yunfan Wu}

Guangdong Second Provincial General Hospital

\section{Guihua Jiang}

Guangdong Second Provincial General Hospital

\section{Research Article}

Keywords: chronic insomnia disorder, DMN, FC, locus coeruleus, seed-based analysis.

Posted Date: January 17th, 2022

DOI: https://doi.org/10.21203/rs.3.rs-1230181/v1

License: (c) (i) This work is licensed under a Creative Commons Attribution 4.0 International License.

Read Full License 


\section{Abstract}

In recent years, people have gained a profound understanding of chronic insomnia disorder (CID), but the pathophysiological mechanism of CID is still unclear. There is some evidence that the locus coeruleus (LC) is involved in the regulation of wakefulness in CID, but there have been few studies using brain functional imaging. The purpose of this study was to evaluate the resting-state functional connectivity (FC) between the LC and other brain voxels in CID and whether these abnormal FC are involved in the regulation of wakefulness. A total of 49 patients with chronic insomnia disorder and 47 healthy controls (HC) matched for gender, age, and education were examined with rs-fMRI in this study. The LC was selected as the region of interest, and then seed-based analysis was conducted on the LC and other voxels to obtain the brain regions with abnormal FC. The correlation between the FC value of the abnormal connection area and the clinical scale score was analyzed. Compared with the HC, the FC between the LC and right precuneus, right posterior cingulate cortex, left middle temporal gyrus, left calcarine, and right superior orbitofrontal cortex was significantly enhanced ( $p<0.05$, FDR correction), and the functional connectivity signal value between the locus coeruleus and left middle temporal gyrus was positively correlated with the Self-Rating Depression Scale $(p=0.021)$. The abnormal FC between the LC and multiple brain regions may contribute to a better understanding of the neurobiological mechanism of CID.

\section{Introduction}

Chronic insomnia disorder (CID) is an independent psychiatric syndrome that involves difficulties in falling or staying asleep that last for at least three months [1, 2].CID affects an estimated $5.8-20.0 \%$ of the population [3] .Persistent insomnia symptoms not only reduce the quality of daily life and affect work efficiency [4] but also lead to depression, anxiety, and even death [5]. Despite the high prevalence and the negative socio-economic impacts of CID, its neurobiological causes and consequences are still elusive.

The locus coeruleus (LC) is considered to play a potentially causal role in sleep and insomnia, particularly in the regulation of the arousal state [6]. The development of neuroimaging techniques has provided a new avenue of research on detecting neurobiological alterations of the LC associated with $\operatorname{CID}[7,8]$. A recent study based on task-state functional MRI in insomnia found that, from no insomnia complaints to major insomnia symptoms, LC activity (assessed by cortical arousal as a proxy variable) increased gradually [9].Evidence from animal model experiments showed that the activity of the LC promoted sensory-evoked awakenings from sleep[10] and reduced non-rapid eye movement (NREM) sleep in sleep disorders [11]. One study in human subjects with a chronically implanted stimulating electrode placed in the region of the LC found that electrical stimulation of the LC produced a profound disruption of sleep patterns in contrast to the normal sleep patterns [12]. Therefore, the LC, as an indispensable nucleus in arousal regulation, may be a breakthrough point to supplement and explain the neurobiological mechanism underlying insomnia. 
Resting-state functional magnetic resonance imaging (rs-fMRI) is superior to other fMRI techniques because of its advantages of simple signal acquisition, small workload of patients, and effective identification of functional areas of different patients [13]. Resting-state functional connectivity (FC) analysis is one of the most effective methods for studying the pathophysiological mechanism of neurologic and psychiatric diseases. FC can use seed-based analysis to select ROls and correlate the average blood oxygenation level-dependent (BOLD) time process of voxels in these ROls with the time process of all other voxels in the brain [14]. A study comparing the functional connectivity of the LC and ventral tegmental area/substantia nigra pars compacta (VTA/SNc) in a healthy population using seed analysis found that LC and VTA/SNc shared negative connectivity to the precuneus, which was consistent with the function of the default mode network (DMN) inactivated under environmental stimulation [15]. Furthermore, performing FC seed analyses by correlating the average LC time course against the time courses of all voxels in the brain, a study of site-specific pharmacological manipulations in healthy volunteers revealed that the posterior cingulate cortex (PCC, the hub region of the DMN) was disconnected from the LC during the altered arousal state and regained connectivity to the LC during the recovery state [16]. This study showed that the functional connectivity of the LC to the PCC, thalamus, and caudate nucleus is related to the level of arousal. However, it is unclear whether the FC between the $\mathrm{LC}$ and DMN is involved in arousal regulation in CID patients.

The aim of this study is to investigate LC-based FC patterns in CID patients and further explore the relationships between the abnormal FC and clinical symptoms. Based on a previous functional neuroimaging study on LC, we hypothesize that the brain regions showing alterations of LC-based FC in $\mathrm{CID}$ are located in DMN regions. The specific alterations of FC in patients with CID will deepen our understanding of the neurobiological mechanism of CID.

\section{Methods}

\section{Subjects}

This prospective study was approved by the ethics committee of Guangdong Second Provincial General Hospital, and all of the participants provided written informed consent after they were provided a complete description of the study. Forty-nine participants with chronic insomnia (21 men; mean \pm standard deviation age, 39.27 years \pm 11.00 ) were recruited from Guangdong Second Provincial General Hospital.

The inclusion criteria for CID participants were as follows: (a) all of the patients had to meet the Diagnostic and Statistical Manual 5 criteria for diagnosis of CID; (b) participants complained of difficulty falling asleep, staying sleep, or waking up prematurely for at least 3 months; (c) participants had no other sleep disorders, such as hypersomnia, parasomnia, or sleep-related movement disorders, or other psychiatric disorders; (d) participants were under 60 years old; (e) participants did not take any psychotropic substances for at least 2 weeks before or during the study; and (f) participants were righthand dominant as assessed using the Edinburgh Handedness Inventory. The exclusion criteria were as 
follows: (a) participants had an abnormal signal in any region of the brain, which was verified by conventional T1-weighted or T2-fluid-attenuated inversion recovery MR imaging; (b) the insomnia disorder was caused by organic disease or severe mental disease that was secondary to depression or generalized anxiety; (c) other sleep disorders; and (d) women who were pregnant, nursing, or menstruating. Forty-seven healthy controls (HC, 15 males and 32 females; age $39.85 \pm 8.97$ ) matched for age, gender, and education were recruited from the local community through advertisements. Healthy controls met the following criteria: (a) Insomnia Severity Index score less than 7; (b) no history of swing shifts, shift work, or sleep complaints; (c) no medication or use of substances such as caffeine, nicotine, or alcohol for at least 2 weeks prior to and during the study; (d) no brain injury or previous severe head trauma, which was verified by conventional T1-weighted or T2-fluid-attenuated inversion recovery MR imaging; (e) no history of psychiatric or neurological diseases; and (f) right-hand dominant.

Several questionnaires were completed by all of the study participants to evaluate the sleep quality and mood status. These questionnaires included the Insomnia Severity Index (ISI), the Pittsburgh Sleep Quality Index (PSQI), the Self-Rating Anxiety Scale (SAS), and the Self-Rating Depression Scale (SDS).

\section{MRI Acquisition}

Functional MR imaging was acquired using a 1.5 Tesla MR scanner (Achieva Nova Dual; Philips, Best, Netherlands) at the Department of Medical Imaging of Guangdong Second Provincial General Hospital. Participants were instructed to rest with their eyes closed and remain still without falling asleep.

Functional MR images were acquired in about 10 minutes using a gradient-echo planar imaging (EPI) sequence as follows: interleaved scanning, repetition time/echo time $=2500 \mathrm{~ms} / 50 \mathrm{~ms}$, section thickness $=4 \mathrm{~mm}$, intersection gap $=0.8 \mathrm{~mm}$, matrix $=64 \times 64$, field of view $=224 \mathrm{~mm} \times 224 \mathrm{~mm}$, flip angle $=90^{\circ}$, 27 axial slices, and 240 volumes. After scanning, all of the subjects were asked if they were asleep during the scan. Those subjects who had fallen asleep were excluded.

\section{MRI Preprocessing and Data Analysis}

Preprocessing of the resting-state fMRI data was carried out using the Data Processing Assistant for Resting-State fMRI (DPARSF; Chao-Gan and Yu-Feng, 2010; http://www.restfmri.net), which is based on Statistical Parametric Mapping (SPM12, Wellcome Centre for Human Neuroimaging, http://www.fil.ion.ucl.ac.uk/spm). The first of 10 images points for each participant were removed to eliminate the effects of an uneven magnetic field at the beginning or the discomfort of the test on image quality and results. Subsequently, the resting-state $\mathrm{fMRI}$ data was regulated for the existence of temporal differences between slice timing and realignment. All of the participants had no more than $2.0 \mathrm{~mm}$ of maximal displacement and 2.0 of maximal rotation in any direction. Then, the fMRI data were spatially normalized to a standard template (Montreal Neurological Institute, MNI) and resampled to $3 \mathrm{~mm}^{3}$. Spatial smoothing was performed with a $4 \mathrm{~mm}$ full width at half-maximum Gaussian kernel. Removal of linear trends and filtering with a bandwidth of $0.01-0.1 \mathrm{~Hz}$ were adopted. Finally, nuisance variables, including 24 head motion parameters, white matter, CSF signals, and global signals, were regressed out. 
The LC is located adjacent to the floor of the fourth ventricle in the rostral pons and extends into the midbrain to the level of the inferior colliculi [17]. The seed region of LC location was defined as a sphere with a radius of $3 \mathrm{~mm}$ to ascertain spatial specificity to very small anatomical structures centered on MNI coordinate $(x=-14 ; y=-34 ; z=-28)$. The MNI coordinate of LC was determined according to a paper about the impact of comparison between the habenula $(\mathrm{Hb})$ and the LC [18] with Duvernoy's brainstem atlas [19]. A voxel-wise FC map of the LC was calculated using the correlations between the mean time series of the seed region and remaining voxels within the brain.

\section{Statistical Analysis}

The demographics and questionnaire scores of all of the participants were analyzed using SPSS (version 20; SPSS, Chicago, III). The Wilcoxon rank sum test was used to compare differences in education level, ISI, PSQI, SAS, and SDS, and a two-sample $t$ test was used to compare differences in age, between CID and HC. Differences associated with gender, however, were assessed using chi-squared tests. A t-test was used to explore the difference in functional connectivity between patients with CID and HC. The threshold of the correction cluster level was set at $p<0.05$, which was considered to be a significant difference. Multiple comparison correction was performed using a Gaussian random field (GRF) ( $p$-voxel $<0.001, p$ cluster < 0.05).

Then, the value of the functional connectivity of each brain region with a significant group effect was extracted from the functional connectivity map and correlated with the PSQI, SAS, SDS, and ISI scores using Spearman correlation analysis.

\section{Results}

The demographic and clinical characteristics of the participants in this study are presented in Table 1. There was no statistically significant difference in gender $(p=0.27)$, age $(p=0.11)$, or education $(p=$ 0.24 ) between the CID and the healthy control groups. The PSQI, SAS, SDS, and ISI scores of the CID group were significantly higher than those of the HC group (all $p<0.001$ ). The $p$ value of gender was obtained using the chi-square test. The $p$ value of age was obtained using the two-sample $t$ test. The $p$ values of ISI, PSQI, SAS, and SDS were obtained using the Wilcoxon rank sum tests. 
Table 1

Demographic and clinical characteristics of CID and HC

\begin{tabular}{|c|c|c|c|}
\hline & \multirow{2}{*}{$\begin{array}{l}\text { CID } \\
(n=49)\end{array}$} & \multirow{2}{*}{$\begin{array}{l}\mathrm{HC} \\
(n=47)\end{array}$} & \multirow[t]{2}{*}{ P Value } \\
\hline & & & \\
\hline Gender (M/F) & $21 / 28$ & $15 / 32$ & 0.27 \\
\hline Age $(y)$ & $39.27 \pm 11.11$ & $39.85 \pm 9.07$ & 0.11 \\
\hline Duration (mo) & $31.67 \pm 52.00$ & $\mathrm{~N} / \mathrm{A}$ & $\mathrm{N} / \mathrm{A}$ \\
\hline Education (y) & $9.33 \pm 5.88$ & $8.34 \pm 4.43$ & 0.24 \\
\hline$|S|$ & $19.67 \pm 3.23$ & $7.17 \pm 2.61$ & $<0.001$ \\
\hline PSQI & $12.80 \pm 3.31$ & $5.21 \pm 2.81$ & $<0.001$ \\
\hline SAS & $51.78 \pm 10.16$ & $42.02 \pm 6.20$ & $<0.001$ \\
\hline SDS & $55.10 \pm 8.67$ & $39.89 \pm 9.31$ & $<0.001$ \\
\hline
\end{tabular}

The comparisons of voxel-wise LC FC between CID and HC are shown in Table 2, Figure 1, and Figure 2. Compared with the HC group, the CID group had enhanced FC with LC in the following brain regions: right precuneus (Precuneus_R), right posterior cingulate cortex (Posterior Cingulate_R), middle temporal gyrus (Temporal_Mid_L left), calcarine (Calcarine_L left), and right superior orbitofrontal cortex (ORBsup.R) (Table 2; Figure 1).

Table 2

Between-group Differences (CID-HC) for Functional Connectivity Strength

\begin{tabular}{|c|c|c|c|c|c|}
\hline \multirow[t]{2}{*}{ Brain Regions } & \multicolumn{3}{|c|}{ MNI Coordinates (Peak) } & \multirow{2}{*}{$\begin{array}{l}\text { Cluster Size } \\
\text { (Voxels) }\end{array}$} & \multirow{2}{*}{$\begin{array}{l}T \\
\text { Values } \\
\text { (Peak) }\end{array}$} \\
\hline & $x$ & $\mathrm{Y}$ & $\mathbf{Z}$ & & \\
\hline Temporal_Mid_L & -51 & -54 & 3 & 20 & 4.88 \\
\hline Calcarine_L & -6 & -87 & -9 & 24 & 4.83 \\
\hline Posterior Cingulate_R & 9 & -60 & 9 & 18 & 4.86 \\
\hline Precuneus_R & 8 & -54 & 65 & 22 & 4.28 \\
\hline ORBsup_R & 25 & -69 & 39 & 16 & 4.28 \\
\hline
\end{tabular}


Correlation analysis showed that the FC value between the LC and the Temporal_Mid_L in CID was negatively correlated with the ISI score in the CID group $\left(r^{2}=0.028 ; p=0.022\right)$ but positively correlated with SDS $\left(r^{2}=0.090 ; p=0.021\right)$ (Figure 3$)$. In addition, the FC value between the $L C$ and the Precuneus_R was negatively correlated with ISI $\left(r^{2}=0.138 ; p=0.012\right)$ and PSQI $\left(r^{2}=0.087 ; p=0.022\right)$, respectively (Figure 4).

\section{Discussion}

The current study employed resting-state fMRI to evaluate the alterations of LC-based FC in patients with CID. Compared to the HC group, we found that patients with CID showed increased LC-based FC mainly in the hub regions of the DMN (including the left MTG, right PCC, and right precuneus), left calcarine cortex, and right OFC. In addition, altered FC was related to patient neuropsychological performance.

Furthermore, we found that the FC values of the LC and DMN (including left MTG, right PCC, and right precuneus) were significantly correlated with sleep parameters (including PSQI, SDS, and ISI). The findings of this study may contribute to a better understanding of the neurobiological mechanisms of CID in arousal regulation abnormalities and behavioral or cognitive impairments.

In the present study, the CID group exhibited stronger FC between the LC and left MTG, right PCC, and right precuneus. These brain regions belong to the DMN or overlap with the DMN. The precuneus is the central hub of the DMN $[17,20]$. The DMN, which is considered to play an important role in the maintenance of consciousness, is one of the most important networks involved in insomnia. It has been found to have an abnormal functional status in both sleep deprivation and insomnia patients, and it is obviously related to sleep quality [21-23]. Previous studies have used some brain regions as seed regions to explore the brain functional connectivity in patients with CID [20,24, 25]. These studies mainly found decreased FC between the seed regions and hub regions of DMN. According to the results, the authors speculated that the functional changes of DMN may be related to insomnia symptoms. Lee et al. [24] used the basal ganglia nucleus as the main seed region to explore the resting-state FC between subcortical regions in insomnia patients and changes following cognitive-behavioral therapy for insomnia (CBTi). The difference between our experiment and previous research is that this study used LC as the seed region, but it also found that the FC between the LC and DMN was increased, which is consistent with previous research findings of FC using seed point analysis. The results of this experiment may further support the hyperarousal hypothesis of insomnia. The regulatory role of the LC in sleep is increasingly recognized. The LC is the main source of norepinephrine. The LC-norepinephrine (LC-NE) system can play an important role in the regulation of arousal/behavioral state, and it is considered to be very important in the regulation of arousal level. Importantly, the overactivity of this system will increase the level of arousal and amplify the emotional response to stress, which will lead to a series of symptoms of hyperexcitability $[26,27]$. Insomnia is one such symptom. Recently, a pharmacological study also showed that the abnormal FC between the LC and PCC (an important node of DMN) is considered to be related to the level of arousal. The study used a potent sedative to induce low-arousal states in healthy subjects and found decreased FC between the LC and the default network key node PCC in the low-wake 
state, while the connection between the two was restored during the recovery state [16]. We speculate that the enhanced FC between the LC and DMN in CID patients may be related to the level of arousal.

In addition, correlation analysis showed that the functional connection signal value between the LC and the left middle temporal gyrus was negatively correlated with the ISI score, and the FC value between the LC and right precuneus was negatively correlated with ISI and PSQI scores; in other words, the LC-DMN FC signal value is negatively correlated with PSQI and ISI scores. This may indicate that the higher the synchronization between these areas and the LC, and the lower the degree of insomnia in patients with insomnia, the better the quality of sleep. This relationship may adopt a compensation mechanism and may further indicate that the arousal promotion mechanism of these patients is complete. At the same time, this study also found that the FC value between the LC and MTG was positively correlated with SDS, indicating that in the CID, the more significant such abnormal FC was, the more serious the degree of depression was. Studies have shown that the severity of insomnia is positively correlated with the degree of depression [28]. Our results suggest that the FC signal value of the LC and MTG may be used as a neurobiological indicator of the severity of insomnia, which needs further verification in future studies.

Increased FC between the LC and left calcarine cortex was found in our study on patients with CID. The calcarine cortex is the concentrated area of the primary visual cortex, which may be related to the greater perceptual advantage of emotional stimulation [29]. In terms of structural and functional magnetic resonance studies, the calcarine cortex (visual cortex) is increasingly considered to be related to the mechanism of insomnia. Recently, a study of changes in cortical structural connections with MRI-based morphometric correlation analysis found that cortical thickness changes between sensory areas (mainly visual, primary auditory, and olfactory cortex) and motor areas in patients with insomnia are often related to sleep quality [30]. A study using the primary visual cortex, auditory cortex, olfactory cortex, and auxiliary motor cortex as ROls, and using seed FC to explore the correlation between insomnia-related complaints and the FC of sensorimotor regions in healthy people, found that the connectivity between the primary visual cortex, auxiliary motor area, and primary auditory region was increased in those who reported difficulty in falling asleep [31]. Such findings are consistent with the hyperarousal model of insomnia, which may maintain arousal and increase unnecessary sensory consciousness, leading to difficulty in falling asleep or easy wakeup from sleep. These studies found that there are structural and functional MRI abnormalities in the calcarine cortex (visual cortex) in patients with insomnia. It is believed that excessive cortical activation leads to the increase of sensory and information processing, thus hindering the ability to start or maintain sleep. These results may indicate that insomnia is related to the hyperarousal of the central nervous system. In our study, we also found abnormal FC between the LC and the calcarine cortex (visual cortex), which is mostly consistent with previous studies. We speculate that the increased activity level of the visual cortex is involved in the mechanism of insomnia. Cells in the LC are the only source of norepinephrine NE in the brain and can change their discharge rate according to the alertness state [26].Animal experiments have confirmed that the LC projects bilaterally to the visual cortex on the anatomical level [32]. Moreover, NE in the forebrain (including the visual cortex) was found to be changed after one week of sleep deprivation in cats, which is speculated that the plasticity of the visual cortex is related to NE change in insomnia deprivation[33]. In other words, the plasticity of the 
visual cortex and the change of LC-NE may be related to insomnia, especially to the hyperarousal hypothesis $[34,35]$. Based on previous studies, our findings confirm abnormal FC in LC and the calcarine cortex (visual cortex), which may provides evidence for the hyperarousal hypothesis of insomnia.

In this study, we also found that, compared with HC, CID patients had stronger FC between the LC and OFC. The OFC, a part of the prefrontal lobe, is involved in the integration and processing of internal and external environmental information, emotional regulation, and episodic memory extraction [36]..Joo et al. found significantly smaller volumes of gray matter in the left orbitofrontal gyrus compared with healthy controls, which may be related to the initiation or maintenance of sleep [37]. A study using the restingstate FC method of voxel-mirrored homotopic connectivity (VMHC) analysis and seed analysis to explore the correlation between insomnia patients and the anterior cingulate cortex found increased FC between the left ACC and right thalamus as well as between the right ACC and left orbitofrontal cortex, indicating that insomnia involves disturbance of sleep-wake regulation [25]. The results show that OFC is abnormal in structure and function in insomnia patients. The prefrontal cortex is considered to be involved in the regulation of sleep and wakefulness. Abnormal FC in the OFC may lead to sleep and wakefulness disorders. However, our study also showed increased FC between the LC and OFC in patients with insomnia compared to healthy controls. This is almost consistent with the findings of previous studies, and we believe that OFC dysfunction may to some extent support the imbalance of arousal regulation in chronic insomnia, which needs to be confirmed by further studies. More importantly, a recent animal study found that the noradrenaline level in the prefrontal cortex, one important region that receives targeted projections from LC neurons, declined after extended prolonged wakefulness, suggesting that the LC cannot preserve noradrenaline release for long periods of time [38]. Based on the above study, we also speculate that the excessive maintenance of wakefulness in patients with insomnia leads to the hyperactivity of the LC, which in turn affects the NE level of the OFC and leads to the disruption of functional connectivity between the LC and OFC [39]. However, at present, this is only a conjecture, and longitudinal research is needed to understand the process more comprehensively.

There are several limitations to be solved in this study. First, the sample size is relatively small,which may weaken the statistical power of detecting differences between groups in some of our measures.Second, an important limitation of this study is that these results are limited to subjective measures of insomnia rather than objective measures. We did not use polysomnography (PSG) to diagnose insomnia, so we can not objectively rule out sleep wake disorders such as obstructive sleep apnea (OSA) and restless legs syndrome. Therefore, the contribution of OSA to brain changes may be underestimated. However, we screened these sleep wake disorders by PSQI questionnaire. Although such an exclusion method is not as accurate as PSG, it may be able to objectively exclude most non-potential OSA. We need to further use objective diagnostic methods to exclude the existence of other sleep wake disorders. Third, considering the small size of the $L C$, we used a very small radius $(3 \mathrm{~mm})$ for the selected sphere, rather than a larger radius, to be as accurate as possible. In addition, considering that the deeper structure of the brainstem is less accurate when choosing the normalization criteria provided by our software, and there is no specific brainstem standardization [40], the small ROI of the LC with a small radius was chosen to make sure that the most normalized parts were captured., the LC, as part of the ascending arousal system, is influenced 
by internal arousal and encoded significantly by noradrenaline [41], and the arousal system has also recently been shown to be affected by individual heart rate variability (HRV) [42]. However, we did not record the heart rate and respiration during $\mathrm{fMRI}$ scanning, and the heart rate effect in this study was not regressed out during the preprocessing steps. This effect of heart rate variability needs to be considered in future research.

\section{Conclusions}

- our study showed that CID patients have abnormalities in seed-based FC between the LC and other brain regions (especially DMN) compared with healthy controls. Our findings suggest that enhanced $\mathrm{FC}$ in the LC is involved in the pathology mechanism of CID.

\section{Declarations}

\section{Acknowledgments}

No.

\section{Author Contributions}

Author contributions included conception and study design (Jiang, Ma and Li), data collection or acquisition ( $\mathrm{Li}$, Huang and Lan), statistical analysis ( $\mathrm{Li}$ and Yang), interpretation of results (Li, Huang, Lan and Liu), drafting the manuscript work or revising it critically for important intellectual content (All authors) and approval of final version to be published and agreement to be accountable for the integrity and accuracy of all aspects of the work (All

authors).

\section{Funding Sources}

This study was funded by grants from the National Natural Science Foundation of China (Grant Nos. 81771807 and 81901729), the Science and Technology Planning Project of Guangzhou (Grant No. 202002030234) and National Science Foundation for Young Scientists of China (Grant No. 82001792).

\section{Conflict of Interest}

We declare that we have no financial and personal relationships with other people or organizations that can inappropriately influence our work, there is no professional or other personal interest of any nature or 
kind in any product, service and/or company that could be construed as influencing the position presented in, or the review of.

\section{Data availability statement}

The data that support the findings of this study are available on request from the corresponding author. The data are not publicly available due to privacy or ethical restrictions.

\section{References}

1. Spiegelhalder, K., Regen, W., Baglioni, C., Nissen, C., Riemann, D., \& Kyle, S. D. (2015). Neuroimaging insights into insomnia. Current neurology and neuroscience reports, 15(3), 9. https://doi.org/10.1007/s11910-015-0527-3

2. Li, Z., Chen, R., Guan, M., Wang, E., Qian, T., Zhao, C. ... Li, Y. (2018). Disrupted brain network topology in chronic insomnia disorder: A resting-state fMRI study. Neurolmage. Clinical, 18, 178-185. https://doi.org/10.1016/j.nicl.2018.01.012

3. Bjorøy, I., Jørgensen, V. A., Pallesen, S., \& Bjorvatn, B. (2020). The Prevalence of Insomnia Subtypes in Relation to Demographic Characteristics, Anxiety, Depression, Alcohol Consumption and Use of Hypnotics. Frontiers in psychology, 11, 527. https://doi.org/10.3389/fpsyg.2020.00527

4. Taylor, D. J., Lichstein, K. L., Durrence, H. H., Reidel, B. W., \& Bush, A. J. (2005). Epidemiology of insomnia, depression, and anxiety. Sleep, 28(11), 1457-1464. https://doi.org/10.1093/sleep/28.11.1457

5. Li, S., Tian, J., Bauer, A., Huang, R., Wen, H., Li, M. ... Jiang, G. (2016). Reduced Integrity of Right Lateralized White Matter in Patients with Primary Insomnia: A Diffusion-Tensor Imaging Study. Radiology, 280(2), 520-528. https://doi.org/10.1148/radiol.2016152038

6. España, R. A., Schmeichel, B. E., \& Berridge, C. W. (2016). Norepinephrine at the nexus of arousal, motivation and relapse. Brain research, 1641(Pt B), 207-216.

https://doi.org/10.1016/j.brainres.2016.01.002

7. Gehrman, P., Sengupta, A., Harders, E., Ubeydullah, E., Pack, A. I., \& Weljie, A. (2018). Altered diurnal states in insomnia reflect peripheral hyperarousal and metabolic desynchrony: a preliminary study. Sleep, 41(5), zsy043. https://doi.org/10.1093/sleep/zsy043

8. Chapman, J. L., Comas, M., Hoyos, C. M., Bartlett, D. J., Grunstein, R. R., \& Gordon, C. J. (2018). Is Metabolic Rate Increased in Insomnia Disorder? A Systematic Review. Frontiers in endocrinology, 9, 374. https://doi.org/10.3389/fendo.2018.00374

9. Wassing, R., Lakbila-Kamal, O., Ramautar, J. R., Stoffers, D., Schalkwijk, F., \& Van Someren, E. (2019). Restless REM Sleep Impedes Overnight Amygdala Adaptation. Current biology: CB, 29(14), 23512358e4. https://doi.org/10.1016/j.cub.2019.06.034 
10. Hayat, H., Regev, N., Matosevich, N., Sales, A., Paredes-Rodriguez, E., Krom, A. J. ... Nir, Y. (2020). Locus coeruleus norepinephrine activity mediates sensory-evoked awakenings from sleep. Science advances, 6(15), eaaz4232. https://doi.org/10.1126/sciadv.aaz4232

11. Koh, K., Hamada, A., Hamada, Y., Yanase, M., Sakaki, M., Someya, K. ... Sakai, H. J. N. L. (2015). Possible involvement of activated locus coeruleus-noradrenergic neurons in pain-related sleep disorders. 589200-206

12. Kaitin, K. I., Bliwise, D. L., Gleason, C., Nino-Murcia, G., Dement, W. C., \& Libet, B. J. B. P. (1986). Sleep disturbance produced by electrical stimulation of the locus coeruleus in a human subject. 21710716

13. Smitha, K., Akhil Raja, K., Arun, K., Rajesh, P., Thomas, B., Kapilamoorthy, T., \& Kesavadas, C. J. N. J. (2017). Resting state fMRI: A review on methods in resting state connectivity analysis and resting state networks.1971400917697342

14. Lee, M. H., Smyser, C. D., \& J.S.J (2013). .A.J.o.N. Shimony, Resting-state fMRI: a review of methods and clinical applications. 341866-1872

15. Sheng, Z., Sien, H., Chao, H. H., \& Li, C. S. R., Resting-State Functional Connectivity of the Locus Coeruleus in Humans: In Comparison with the Ventral Tegmental Area/SubstantiaNigra Pars Compacta and the Effects of Age, pp.3413

16. Song, A. H., Kucyi, A., Napadow, V., Brown, E. N., \& Loggia, M. L. (2017). and O.J.J.o.N. Akeju, Pharmacological Modulation of Noradrenergic Arousal Circuitry Disrupts Functional Connectivity of the Locus Ceruleus in Humans.446-417

17. Fransson, P., \& Marrelec, G. J. N. (2008). The precuneus/posterior cingulate cortex plays a pivotal role in the default mode network: Evidence from a partial correlation network analysis. 421178-1184

18. Erpelding, N., Sava, S., Simons, L. E., \& Lebel, A. (2014). and D.J.J.o.N. Borsook, Habenula functional resting-state connectivity in pediatric CRPS. 111239--247

19. Naidich, T. P., Duvernoy, H. M., Delman, B. N., Sorensen, A. G., \& Haacke, E. M. (2009). Duvernoy's Atlas of the Human Brain Stem and Cerebellum. Springer Vienna

20. Yan, C. Q., Wang, X., Huo, J. W., \& Zhou, P. (2018). and Q.Q.J.F.i.N. Liu, Abnormal Global Brain Functional Connectivity in Primary Insomnia Patients: A Resting-State Functional MRI Study. 9

21. Dong, X., Qin, H., Wu, T., Hu, H., Lei, X. J. B., \& Behavior (2018). Rest but busy: Aberrant resting-state functional connectivity of triple network model in insomnia. $8 \mathrm{e} 00876$

22. Yu, S., Guo, B., Shen, Z., Wang, Z., Yu, K., \& Hu, Y. (2018). and F.J.J.o.P.R. Feng, The imbalanced anterior and posterior default mode network in the primary insomnia.S002239561830181X

23. Havas, J. A. D., Parimal, S., Soon, C. S., \& Chee, M. W. L. J. N. (2012). Sleep deprivation reduces default mode network connectivity and anti-correlation during rest and task performance. 5917451751

24. Lee, Y. J. G., Kim, S., Kim, N., Choi, J. W., Park, J., Kim, S. J. ... Lee, Y. J. J. N. C., Changes in subcortical resting-state functional connectivity in patients with psychophysiological insomnia after cognitivebehavioral therapy. 17115-123

Page $12 / 16$ 
25. Chao-Qun, Y., Cun-Zhi, L., Xu, W., Jian-Wei, H., Ping, Z., Shuai, Z. ... Zhong-Yan, W. (2018). and L.J.F.i.A.N. Qing-Quan, Abnormal Functional Connectivity of Anterior Cingulate Cortex in Patients With Primary Insomnia: A Resting-State Functional Magnetic Resonance Imaging Study. 10167

26. Yamamoto, K. I., Shinba, T., Yoshii, M. J. P., \& Neurosciences, C. (2014). Psychiatric symptoms of noradrenergic dysfunction: a pathophysiological view. 68

27. C.W.J.B.R, R., \& Berridge (2008).Noradrenergic modulation of arousal.581-17

28. Gupta, R., V.J.I.J.o.P, M., \& Lahan (2012). 33,2, Insomnia Associated with Depressive Disorder: Primary, Secondary, or Mixed? 33123-128

29. Su, H., Zuo, C., Zhang, H., Jiao, F., Zhang, B., Tang, W. ... Guan, Y. (2018). S.J.Q.I.i.M. Shi, and Surgery, Regional cerebral metabolism alterations affect resting-state functional connectivity in major depressive disorder. 8 910-924

30. Lu, Z., Enfeng, W., Xiaoqi, Z., Sherif, K., Budhachandra, K., Hongju, Z. ... Dapeng, S. J. B. R. I. (2015). Cortical Structural Connectivity Alterations in Primary Insomnia: Insights from MRI-Based Morphometric Correlation Analysis. (2015) 1-23

31. Killgore, W. D. S., Schwab, Z. J., Kipman, M., Deldonno, S. R., \& Weber, M. J. N. (2013). Insomniarelated complaints correlate with functional connectivity between sensory-motor regions. 24233-240

32. Sato, H., Fox, K., \& Daw, N. W. J. J. N. (1989). Effect of electrical stimulation of locus coeruleus on the activity of neurons in the cat visual cortex. 62946-58

33. Shaffery, J. P., Allard, J. S., \& Manaye, K. F. (2012). and H.P.J.F.i.N. Roffwarg, Selective Rapid Eye Movement Sleep Deprivation Affects Cell Size and Number in Kitten Locus Coeruleus. 369

34. Normand, M., Sthilaire, P., \& Bastien, C. H. J. N. P. (2016). Sleep Spindles Characteristics in Insomnia Sufferers and Their Relationship with Sleep Misperception. (2016) 6413473-6413473

35. Aton, S. J., Seibt, J., Dumoulin, M. C., Coleman, T., \& Shiraishi, M. (2009). M.G. Frank, and O.J.J.P.O. Manzoni, The Sedating Antidepressant Trazodone Impairs Sleep-Dependent Cortical Plasticity. 4e6078-

36. Frith (1999). and D.J.S. C., Interacting Minds-A Biological Basis. 2861692-1695

37. Yeon, J. E., Jin, N. H., Jeong-Sik, K., Lim, K. D., Daeyoung, K., Jin, H. K. ... Bong, H. S. J. S., Brain Gray Matter Deficits in Patients with Chronic Primary Insomnia. 7.

38. Bellesi, M., Tononi, G., Cirelli, C., \& Serra, P. A. J. S. (2016). Region-Specific Dissociation between Cortical Noradrenaline Levels and the Sleep/Wake Cycle. 39143-154

39. Kim, Y., Chen, L., Mccarley, R. W., \& Strecker, R. E. J. B. R. (2013). Sleep allostasis in chronic sleep restriction: The role of the norepinephrine system. 15319-16

40. A, N. I. K., C.T.L. A, \& K.C.H. A (2009). P.S.M.B. C, and M.A.E.J.N. A, In vivo mapping of the human locus coeruleus. 471261-1267

41. Nieuwenhuys, R., Voogd, J., \& Huijzen, C. V. (1988).The Human Central Nervous System, Springer,

42. Chang, C., Metzger, C. D., Glover, G. H., Duyn, J. H., Heinze, H. J., \& Walter, M. J. N. (2013). Association between heart rate variability and fluctuations in resting-state functional connectivity. 6893-104 


\section{Figures}
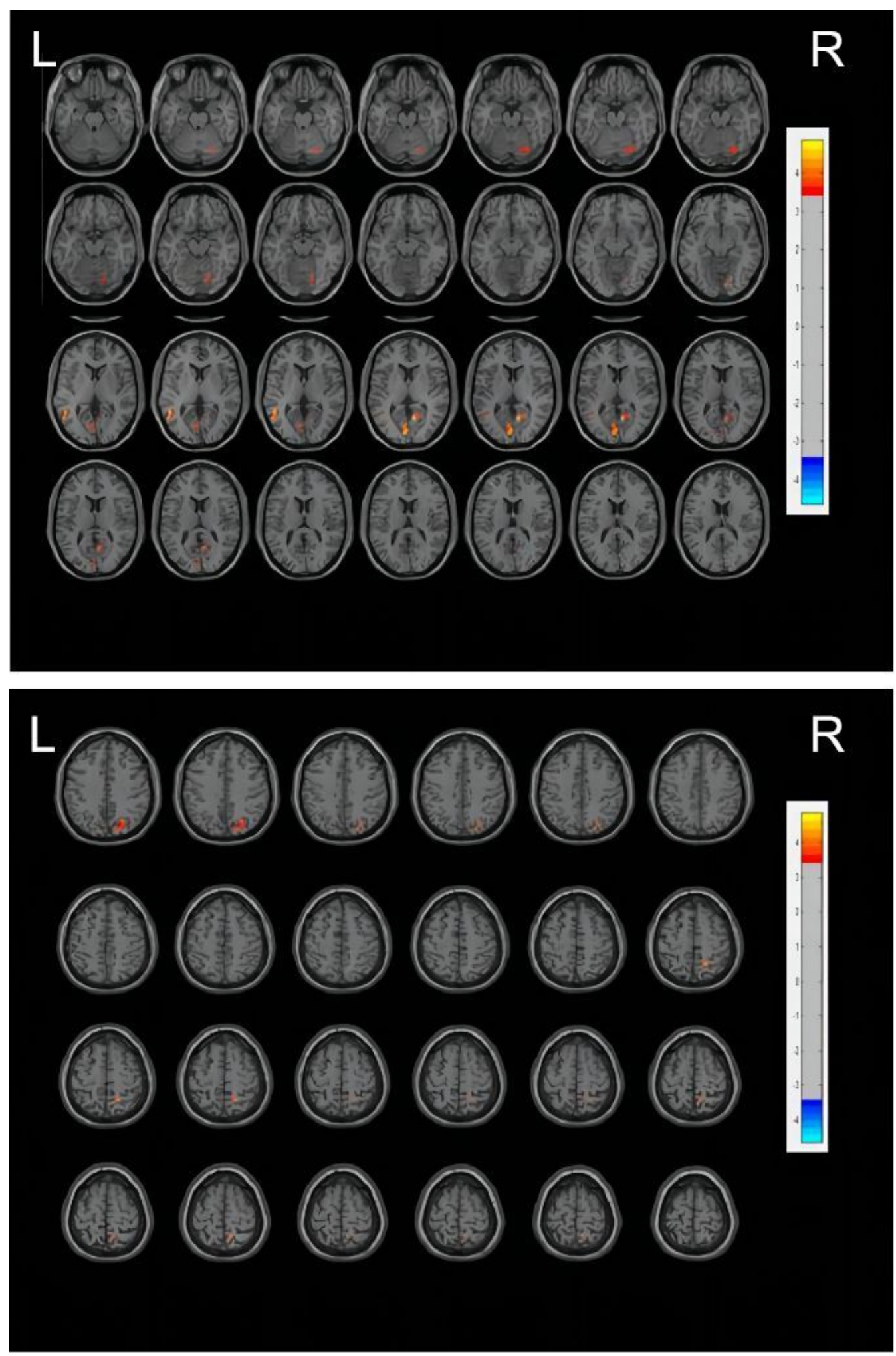

\section{Figure 1}

Functional connectivity differences of the LC between CID patients and healthy controls. L:left; R:right. The threshold of the color bar means the $T$ value. The warm color (right precuneus, right posterior 
cingulate cortex, left middle temporal gyrus, left calcarine, right superior orbitofrontal cortex) represent increased connectivity.

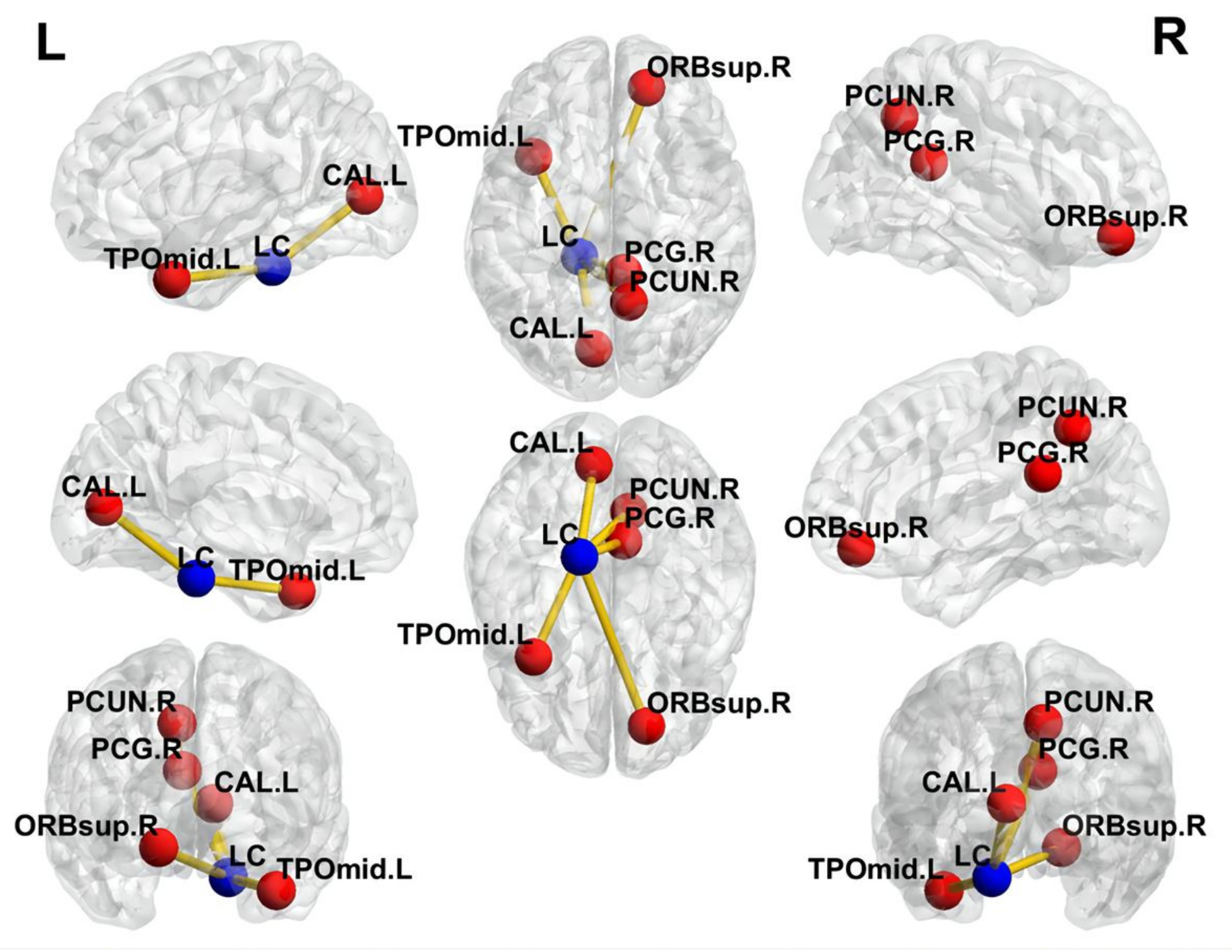

Figure 2

Visualization of brain regions with increased functional connectivity with the locus coeruleus in CID.The figure is marked as an abbreviation in the Anatomical Automatic Labeling (ALL). L:left; R:right;LC:locus Coeruleus ; TPOmid.L: left middle temporal gyrus(MTG);CAL.L: left calcarine; PCG.R: right posterior cingulate cortex (PCC);PCUN.R : right precuneus ; ORBsup.R: right superior orbitofrontal cortex (OFC). The result of the brain regions that the research obtained is Visualized with the BrainNetViewer (Xia.et.al.,2013, http://www.nitrc.org/projects/bnv/) 


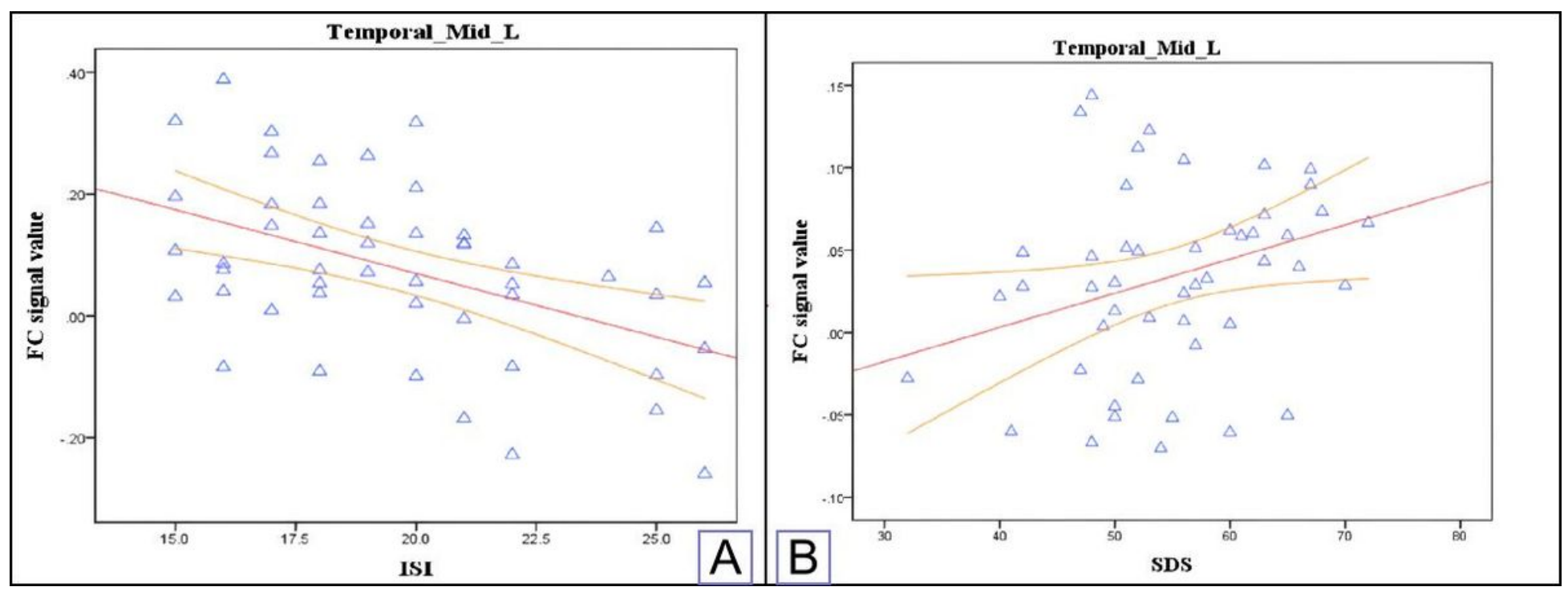

Figure 3

Significant correlations between FC of LC-Temporal_Mid_L and ISI score (Fig.A, $\left.r^{2}=0.028 ; p=0.022\right)$ as well as SDS in CID (Fig.B, $\left.r^{2}=0.090 ; p=0.021\right)$.

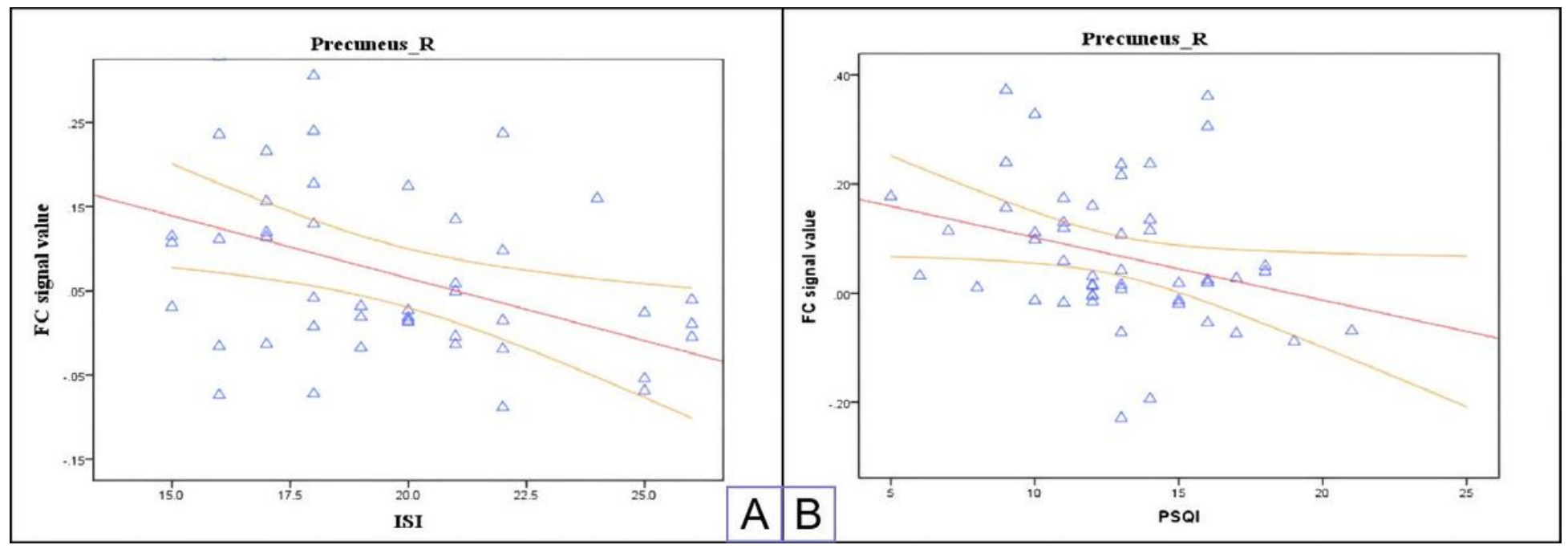

\section{Figure 4}

Significant negative correlations between FC of LC-Precuneus_R and ISI ( Fig.A, $I^{2}=0.138 ; p=0.012$ ) as well as PSQI(Fig.B, $r^{2}=0.087 ; p=0.022$ ).

\section{Supplementary Files}

This is a list of supplementary files associated with this preprint. Click to download.

- 1657317173667707BIBChecklist.docx 